P004

\section{GUIDELINES AND PERFORMANCE}

Daniel Keenan.

\section{0:1136/bmjgs-2013-002293.4}

The Guidelines and performance measures allow us to reduce variation in the provision of care and to provide reassurance to all that care is optimum.

In England we continue to look at clinical performance and have focused of the three main tenants of quality; clinical effectiveness, safety and patient experience from institution down to individual. Institutional effectiveness has been looked at in relation to targets which are "must do", such as primary angioplasty times (previously thrombolysis) and performance against standards such as time to scan for stroke. Next come such measures as the percentage of patients admitted to a dedicated stroke unit and the use of cognitive behavioural interventions. In relation to individual performance, we have looked at mortality rates following open-heart surgery. This year, under the government's transparency agenda, individual results are to be published from additional national interventional audits. More are to follow and virtually all the national audits and registries will be published with a high degree of granularity in the next few years.

In primary care the quality and outcomes framework, which is a remuneration package for primary care physicians, is used to manage performance and has driven up the quality of the services offered. It is based on a basket of process measures, which are managed by the National Institute for Health and Care Excellence (NICE), and are renegotiated to drive increasing improvement that is evident by improved performance against the indicators.

With recent changes in government policy, increasing interest has focused on the quality of commissioning and measures have been derived from NICE Quality Standards (in effect an amalgamation of guidelines into 12 standards) to assess this. Further, with the introduction in the UK this year of medical revalidation, there is an increased emphasis on individual information to support re licensing.

Overall, therefore, we have a dual track of the increasing development of guidance and the condensation of these into easily manageable standards and the measurement of performance, at many levels, against these standards.

\section{P005 DO GUIDELINES GUIDE THE CLINICAL PRACTICE?}

Sang il Lee.

\section{0:1136/bmjgs-2013-002293.5}

Clinical practice guidelines and performance measures can be used to improve quality of health care. Medical review criteria and performance measures are used to determine the extent to which care has followed specified processes and whether the expected outcomes have been achieved. Recently there are increasing tendencies to release the healthcare organisations' performance to the public and to introduce pay-for-performance in many countries.

In countries where development, dissemination, and implementation of clinical practice guidelines are relatively slow, other interventions such as claims review activities, report cards, and pay-for-performance may have stronger influence on clinical practice in the real world than guidelines. In this presentation issues around the relationship among clinical practice guidelines, performance measures, claims review criteria, and clinical practice in the settings where guideline activities are not so active will be discussed

\section{Plenary 3: Challenges and Solutions for Updating Guidelines}

\section{P006 UPDATING PRACTICE GUIDELINES}

Paul Shekelle (USA).

\section{0:1136/bmjgs-2013-002293.6}

A challenge facing all guideline developers is how to keep their guidelines up-to-date. The practice of using a fixed interval to update guidelines, such as every 3 or 5 years, may not be efficient in terms of resources or keep fast-moving guidelines sufficiently up-to-date. I will present a conceptual framework for considering when guidelines need updating, that includes periodic surveillance. Next I will present the results of a programme of surveillance of a large number of systematic reviews, a conceptually similar process. I will then relate our experience with implementing a programme of surveillance for guidelines produced by the American College of Physicians. I will conclude with thoughts and speculations about the future directions for surveillance and updating of guidelines

\section{P007 KEEPING A PROGRAMME OF CLINICAL GUIDELINES UP-TO-DATE}

Roberta James

10:1136/bmjqs-2013-002293.7

Over the last 20 years, SIGN has published 132 guidelines. Of the 68 extant guidelines only 15 are under 3 years old. SIGN is committed to providing evidence based clinical practice guidelines to help accelerate the translation of new knowledge into action to meet our aim of reducing variations in practice, and improving patient-important outcomes.

SIGN has an established process of scoping, consulting on, prioritising and updating published guidelines. This process, however, is time and resource intensive and has been based heavily on the age of the guideline and the emergence of new evidence rather than on any real need from guideline users.

A full review of a guideline is equivalent to developing a new topic and takes 2-3 years. Some alternative approaches that SIGN has developed for keeping guidelines up to date in a more timely fashion include:

- a selective update based only on those key questions underpinning a guideline that are shown to identify new evidence that would change recommendations. This process takes around 15 months and topics are scoped and prioritised;

- a living guideline that is scoped and updated every year;

- a small change affecting one or two recommendations that is highlighted by a guideline user or initiated by a change in licensing, legislation or local healthcare policy. This process takes around 6 months, is more reactive and driven by guideline user need but may only be applicable to newer guidelines.

This presentation will describe the risks, benefits, success and challenges in adopting these approaches. The key lessons learned include the need to: 
- clarify the need for the update

- scope the evidence base and define the breadth of the remit

- follow established processes and work within existing methodology

- manage guideline development group expectations.

\section{P008 KEEPING CANCER GUIDELINES CURRENT USING A WIKI APPROACH}

Ian Olver (Australia)

10:1136/bmjqs-2013-002293.8

Updating written guidelines regularly is difficult and expensive. New evidence in cancer treatment is published frequently. Guideline booklets are also difficult to disseminate widely and stakeholder feedback is mainly pre-publication. To address these issues, Cancer Council Australia developed a web-based wiki platform for guidelines. Only invited expert authors can write in the wiki guidelines, but any stakeholder can comment upon them at any time. The key steps in guideline development were integrated with the wiki capability. An expert group, whose competing interests are documented, were identified, the key clinical questions and search strategies were developed for each question and literature searches recorded on the wiki. An online literature screening and critical appraisal process was developed. Evidencebased recommendations were formulated and evidence tables automatically generated. The stakeholders were invited to comment online. Web analytics monitored usage. The writers remain engaged to appraise new papers and update the guideline rapidly as necessary. All previous versions could be accessed. We evaluated lung cancer treatment guidelines developed on the wiki, where 22 authors identified 67 clinical questions. The literature search and screening process resulted in 2035 potentially relevant articles being forwarded for detailed methodological evaluation with another 571 added through snowballing and other methods. To fine-tune the initial draft content, the working party used the wiki to exchange 156 internal comments in 9 weeks. Of 1055 visits in a 30 day initial consultation period 487 were targeted by email and 387 found the site by Google searches. Of respondents from 45 countries, most were from Australia (799 visits), New Zealand (60 visits) and the United States (31 visits) Of 38 comments, 31 resulted in edits. A strategy to boost uptake is to write Qstream education modules to accompany the guidelines.

\section{Plenary 4: Developing Implementable Guidelines}

\section{P009 GUIDELINE IMPLEMENTABILITY: LEARNING FROM GREAT THINKERS LIKE PICASSO, THE DALAI LAMA AND ANONYMOUS}

Melissa Brouwers (Canada)

\section{0:1136/bmjqs-2013-002293.9}

A tension faced in the guideline enterprise is finding the right balance between development, implementation and evaluation of recommendations. Are developers getting mired in the minutiae of creating the best guideline report? Are implementers choosing the best messages and strategies to optimise utilisation? Are evaluators assessing the most meaningful outcomes or the outcomes that are easiest to measure? Together, are these players in the guideline enterprise optimising what is known in their respective fields and the relationships among each other to create a system for success? In this presentation, we will turn for inspiration to some great thinkers, consider the research environment, and bring in real life examples to address these questions.

\section{P010 DEVELOPING AND EVALUATING COMMUNICATION STRATEGIES TO SUPPORT INFORMED DECISIONS AND PRACTICE BASED ON EVIDENCE (DECIDE) FOR HEALTH PROFESSIONALS}

Pablo Alonso (Spain).

10:1136/bmjqs-2013-002293.10

The DECIDE initiative, building on the work of GRADE, is exploring methods to ensure effective communication of evidence-based recommendations targeted at key stakeholders (healthcare professionals, policymakers, and managers, and patients and the general public). DECIDE will produce strategies for communicating recommendations that are being rigorously evaluated in diverse settings, and it will support the transfer of research into practice in healthcare systems globally. The methodology is an iterative process that includes; brainstorming, user feedback through user testing, surveys, trials and implementation and evaluation in real guidelines. All this is being done across a wide range of healthcare systems in Europe, North America, and other countries.

The work with healthcare professionals is developing three strategies: 1) An electronic multilayered guideline format that presents the essential information that healthcare professionals tell us they need to understand and act on a recommendation; 2) An evidence to recommendation table for users: this is a summary table with the factors for moving from evidence to a recommendation and the justification for each recommendation; 3) A decision aid template to semi-automatically build decision aids linked to guidelines to be used at the point of care. For strategies 1 and 3 DECIDE is also collaborating with the MAGIC programme.

These strategies will be implemented a guideline authoring tool that is being developed from GRADEpro (called the Guideline Development Tool, or GDT), and will also be implementable in other online guideline authoring tools, allowing guideline groups to decide which of these strategies to use when developing their guidelines. The GDT will be able to facilitate the full authoring of a typical guideline and allow the updating of these strategies when needed. Guideline outputs will be made available through multiple platforms (e.g., websites, smartphones and tablets apps).

These tools will help to make guidelines better suited to the information needs of health professionals, policymakers and consumers across diverse healthcare settings in Europe and elsewhere.

\section{P011 SUCCESS AND CHALLENGES FROM OVER 5 YEARS OF THE NATIONAL STROKE FOUNDATION'S STROKELINK PROGRAM. AN EXAMPLE OF A COMPREHENSIVE IMPLEMENTATION PROGRAM LINKING STROKE GUIDELINES TO CURRENT PRACTICE IN AUSTRALIA}

Kelvin Hill (Australia).

10:1136/bmjqs-2013-002293.11 